\title{
Wurzelextrakt als pflanzlicher Immunmodulator
}

Es ist schwierig, die Immunkompetenz in Studien umfassend zu beschreiben, weil sie von vielen Faktoren beeinflusst wird. Hierzu gehören das Alter, Stress und Krankheiten. Deshalb sollte ein geeignetes Messverfahren der Immunkompetenz mehrere Parameter gleichzeitig berücksichtigen.

Mit dem „Scoring of Immunological Vigor" (SIV) wurde ein solches objektives Verfahren entwickelt und mittlerweile mehrfach erprobt. Es beurteilt die folgenden 8 Parameter des erworbenen Immunsystems in 3 Abstufungsgraden ( 3 =hoch, $2=$ mittel und $1=$ niedrig): Gesamtzahl an T-Zellen, naive T-Zellen, $\mathrm{CD}^{+} \mathrm{CD} 28^{+} \mathrm{T}-Z \mathrm{Zl}-$ len, natürliche Killer-Zellen und B-Zellen, das Verhältnis von $\mathrm{CD}^{+} / \mathrm{CD}^{+} \mathrm{T}$-Zellen und der naiven/Gedächtnis-T-Zellen sowie den T-Zell-Proliferativindex (TCPI).

\section{Tongkat Ali in placebo- kontrollierter Studie}

Mithilfe des SIV untersuchte nun eine asiatische Arbeitsgruppe, inwieweit ein standardisierter wässriger Wurzelextrakt aus Eurycoma longifolia (Physta ${ }^{\circledR}$ ), auch als Tongkat Ali bezeichnet, das Immunsystem stärken kann. Hintergrund der Wahl dieses Pflanzenextrakts waren Ergebnisse aus klinischen und In-vitro-Untersuchungen. In diesen früheren Tests zeigte das Präparat Effekte auf den Testosteron- und Muskelstoffwechsel, auf die Erholung nach Erschöpfung sowie anti-

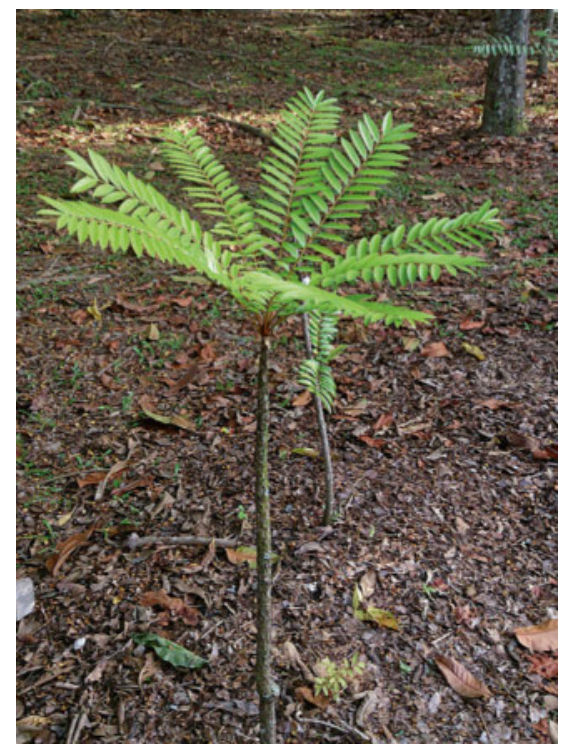

Heimisch in Malaysia und Indonesien: Eurycoma longifolia Jack, ein Bittereschengewächs. (c) Mokkie

oxidative und Antitumoraktivität. Letztere ist vermutlich v.a. auf den Inhaltsstoff Eurycomanon zurückzuführen.

Die 84 Probanden im mittleren Alter von 40-59 Jahren mit niedrigen SIVWerten nahmen über den Zeitraum von 4 Wochen randomisiert entweder eine Kapsel mit 200 mg Eurycoma-longifoliaWurzelextrakt (Verum) oder 200mg Reispulver (Placebo) zu sich und sollten ansonsten ihren normalen Lebensstil beibehalten. Am Ende der Studienzeit wurde der Immunstatus mittels der 8 Parameter ermittelt, die in die Berechnung des SIV eingingen. Es ergab sich in der Verumgruppe ein signifikanter Unterschied der SIV-Werte zwischen Studienbeginn und -ende, während dies in der Placebogruppe nicht der Fall war. Ebenso hatte sich der Immunstatus der Probanden in der Verum- gegenüber der Placebogruppe signifikant verbessert sowie der Wert für das errechnete immunologische Alter. Die untersuchten Laborwerte zur Sicherheit des Pflanzenpräparats unterschieden sich in einigen Punkten zwar innerhalb der Gruppen von Woche $0 \mathrm{zu} 4$, jedoch ohne klinische Auswirkung. Milde Nebenwirkungen traten in beiden Gruppen ohne statistisch relevanten Unterschied auf.

\section{Fazit}

Ein wässriger Extrakt aus der Wurzel von Eurycoma longifolia verbessert über einen Zeitraum von 4 Wochen die Funktion des erworbenen Immunsystems bei Personen mittleren Alters mit reduziertem Immunstatus. Ob sich auch andere Immunparameter durch das Pflanzenpräparat ändern oder auch jüngere Personen davon profitieren, müsste in weiteren Untersuchungen geklärt werden - ebenso wie dessen Wirkung über einen längeren Zeitraum als die untersuchten 4 Wochen.

\section{Ulrike Andres, Ginsheim}

\section{Literatur}

George A, Suzuki N, Abas AB et al. Immunomodulation in middle-aged humans via the ingestion of Physta ${ }^{\circledR}$ standardized root water extract of Eurycoma longifolia Jack - a randomized, double-blind, placebo-controlled, parallel study. Phythother Res 2016; 30: 627-635 